\title{
A THEOREM ON FREE ENVELOPES
}

BY

CHESTER C. JOHN, JR.

\begin{abstract}
The free envelope of a finite commutative semigroup was defined by Grillet [Trans. Amer. Math. Soc. 149 (1970), 665-682] to be a finitely generated free commutative semigroup $F(S)$ with identity and a homomorphism a: $S \rightarrow F(S)$ endowed with certain properties. Grillet raised the following question: does $\alpha(S)$ always generate a pure subgroup of the free Abelian group with the same basis as $F(S)$ ? We prove this is indeed the case. It follows as a result of two lemmas.

Lemma 1: Given a full rank proper subgroup $H$ of a finitely generated free Abelian group $F$ and a basis $X$ of $F$ there exists a surjective homomorphism $f: F \rightarrow Z$ such that $f$ is positive on $X$ and $f_{\mid H}$ is not surjective. Lemma 2: A finitely generated totally cancellative reduced subsemigroup of a finitely generated free Abelian group $F$ is contained in the positive cone of some basis of $F$. The following duality theorem is also proved. Let $S^{*} \simeq$ $\operatorname{Hom}(S, N)$ where $N$ is the nonnegative integers under addition. Then $S \simeq S^{* *}$ if and only if $S$ is isomorphic to a unitary subsemigroup of a finitely generated free commutative semigroup with identity.
\end{abstract}

The free envelope of a finitely generated commutative semigroup $S$ was introduced in [1]. It is a finitely generated free commutative semigroup $F(S)$ with identity together with a homomorphism $\alpha: S \rightarrow F(S)$ endowed with certain properties, in particular any homomorphism $f: S \rightarrow F$, where $F$ is a finitely generated free commutative semigroup with identity, factors, not necessarily uniquely, through $\alpha$.

The following question was raised in [1]: Does $\alpha(S)$ always generate a pure subgroup in the free Abelian group with the same basis as $F(S)$ ? We prove that this is indeed the case. As an application of this result, we prove the following duality theorem (where $S^{*}=\operatorname{Hom}(S, N)$, and $\mathbf{N}$ is the nonnegative integers under addition): $S \simeq S^{* *}$ if and only if $S$ is isomorphic to a unitary subsemigroup of a finitely generated free commutative semigroup with identity. The notation is the same as in [1], in particular, all semigroups are commutative, denoted additively, and identity elements are denoted by 0 .

Recall that a commutative semigroup $S$ is said to be totally cancellative if it is power cancellative (i.e., $n a=n b$ implies $a=b$ for all $n \in \mathbf{N} \backslash\{0\}$ ) and cancellative, and that a totally cancellative semigroup $S$ is said to be reduced if either it has no identity element or its group of units is trivial.

The proof of the main theorem depends on the following lemmas.

Received by the editors November 16, 1973.

AMS (MOS) subject classifications (1970). Primary 20M05, 20M15, 20K15, 20M20, $20 \mathrm{M} 30$. 
LEMMA 1. If $F$ is a finitely generated free Abelian group, $H$ is a subgroup of $F$ of the same rank as $F$, and $x_{1}, x_{2}, \ldots, x_{n}$ is a free generating set of $F$, then there exists a surjective homomorphism $\beta: F \rightarrow Z$ such that:

(1) $\beta_{\mid H}$ is not surjective;

(2) $\beta\left(x_{i}\right)>0$ for $i=1,2, \ldots, n$.

Proof. The proof will proceed by induction on the rank of $F$. If $\operatorname{dim}(F)=$ 1 , then $F \simeq Z$. Thus define $\beta=\theta$ if $\theta\left(x_{1}\right)=1$ and $\beta=-\theta$ otherwise, where $\theta$ is the isomorphism from $F$ to $Z$ above.

Assume the lemma for all free Abelian groups of rank $n-1$, and let $F$ have rank $n$. Since $F / H$ is torsion there exists a smallest positive integer $\boldsymbol{n}_{\boldsymbol{i}}$ such that $n_{i} x_{i} \in H$ for $i=1,2, \ldots, n$.

Let $F^{\prime}$ be the free Abelian group generated by $x_{2}, x_{3}, \ldots, x_{n}$, and let $H^{\prime}=H \cap F^{\prime}$.

Case (1). Assume $H^{\prime}=F^{\prime}$. Then $n_{1}>1$ since $H$ is a proper subgroup of $F$. Let $\beta\left(x_{1}\right)=1$ and $\beta\left(x_{i}\right)=n_{1}$ for $i=2,3, \ldots, n$. Then $n_{1} \mid \beta(h)$ for all $h \in H$. But $\beta$ is surjective since $\beta\left(x_{1}\right)=1$.

Case (2). Assume $H^{\prime} \neq F^{\prime}$.

Since $H^{\prime} \subseteq F^{\prime}$ contains $n_{2} x_{2}, \ldots, n_{n} x_{n}$, it has rank $n-1$ and by the induction hypothesis there exists a surjective homomorphism $\beta^{\prime}: F^{\prime} \rightarrow Z$ such that $\beta_{\mid H^{\prime}}^{\prime}$ is not surjective and $c_{i}=\beta^{\prime}\left(x_{i}\right)>0$ for $i=2,3, \ldots, n$. Let $c$ be the smallest positive integer in $\beta^{\prime}\left(H^{\prime}\right)$. Then $\operatorname{gcd}_{i>1}\left(c_{i}\right)=1$ and $c>1$. Clearly there exists a smallest $k$ such that $0<k<n_{1}$ and $\left(k x_{1}+F^{\prime}\right) \cap H \neq \varnothing$. Thus there exists $\Sigma_{i>1} p_{i} x_{i}$ such that $0<p_{i}<n_{i}$ and $k x_{1}+\Sigma_{i>1} p_{i} x_{i} \in H$.

Let $j=\operatorname{lcm}_{i>1}\left(c_{i}\right)$ and $b=\operatorname{gcd}\left(k, \Sigma_{i>1}\left(c j n_{i}-c_{i} p_{i}\right)\right)$. Define $c_{1}^{\prime}=\left(\Sigma_{i>1} c j n_{i}\right.$ $\left.-c_{i} p_{i}\right) / b$ and $c_{i}^{\prime}=(k / b) c_{i}$ for $i=2,3, \ldots, n$. It is clear that $\operatorname{gcd}_{i>1}\left(c_{i}^{\prime}\right)=1$ and $c_{i}^{\prime}>0$. Define $\beta\left(\sum_{i>1} b_{i} x_{i}\right)=\sum_{i>1} b_{i} c_{i}^{\prime}$. Then $\beta$ is surjective since $\operatorname{gcd}_{i>1}\left(c_{i}^{\prime}\right)=1$ and $\beta\left(x_{i}\right)=c_{i}^{\prime}$.

Let $x=\sum b_{i} x_{i} \in H$. If $b_{1}=0$ then $x \in H^{\prime}$. Thus assume $b_{1} \neq 0$, then $k \mid b_{1}$ by the choice of $k$, say $b_{1}=s k$. Thus $x-s\left(k x_{1}+\Sigma p_{i} x_{i}\right) \in H^{\prime}$. Therefore, $H$ is generated by $H^{\prime}$ and $k x_{1}+\Sigma p_{i} x_{i}$. Hence

$$
\left\langle H^{\prime} \cup\left\{k x_{1}+\sum p_{i} x_{i}\right\}\right\rangle=H .
$$

By the definition of $\beta$ it is clear that for all $r \in H^{\prime}, c \mid \beta(r)$ and $\beta\left(k x_{1}+\right.$ $\left.\sum p_{i} x_{i}\right)=c(k / b) j \sum n_{i}$. Thus $c \mid \beta\left(k x_{1}+\sum p_{i} x_{i}\right)$. Therefore, for all $r \in H$, $c \mid \beta(r)$, and thus the lemma is proved.

In what follows, $\left\langle x_{1}, \ldots, x_{n}\right\rangle$ denotes the subsemigroup (not the subgroup) generated by $x_{1}, \ldots, x_{n}$.

Let $S$ and $T$ be cancellative commutative semigroups, then $G(S)$ denotes the universal group of $S$, and if $f: S \rightarrow T$ is a homomorphism, then $G(f)$ : $G(S) \rightarrow G(T)$ is the induced homomorphism.

LEMMA 2. Let $S$ be a finitely generated totally cancellative reduced semigroup which is embedded into a finitely generated free Abelian group $F$. There exists a basis of $F, x_{1}, \ldots, x_{n}$, such that $S \subseteq\left\langle x_{1}, \ldots, x_{n}\right\rangle$. 
Proof. From [1] there is a homomorphism $f: S \rightarrow \mathbf{N}$ which is positive on $S \backslash\{0\}$. Since $S$ is both commutative and cancellative $f$ can be extended to $G(f): G(S) \rightarrow Z$. Let $G(S)^{d}$ be the divisible hull of $G(S)$. Then $G(f)$ can be extended to $f^{\prime}: G(S)^{d} \rightarrow \mathbf{Q}$. Since divisible groups are injective there is a homomorphism $g: G(F) \rightarrow Q$ such that $g$ is positive on $S \backslash\{0\}$. Let $T=$ $\operatorname{Ker}(g)$, the kernel of $g$. Then $T$ has rank $n-1$ since $g$ has rank $n$, and hence $G(F)$ has a basis $x_{1}, \ldots, x_{n}$ such that $m_{2} x_{2}, \ldots, m_{n} x_{n}$ is a basis of $T$. Since $g(m x)=0$ implies $g(x)=0$, we see that $x_{2}, \ldots, x_{n}$ is a basis of $T$. Replacing $x_{1}$ by $-x_{1}$ if necessary we can also arrange that $g\left(x_{1}\right)>0$. When $x=\sum p_{i} x_{i}$ $\in S \backslash\{0\}$ we have $f(x)=p_{1} f\left(x_{1}\right)>0$. Thus $p_{1}>0$.

Let $\left\{s_{1}, s_{2}, \ldots, s_{k}\right\}$ be a generating set of $S$, and for each $i, s_{i}=\Sigma_{j>1} b_{j}^{i} x_{j}$. Then by the choice of $x_{1}, b_{1}^{i}>0$ for each $i$. Therefore, pick $p \in \mathbf{N}$ such that $b_{j}^{i}+p b_{1}^{i}>0$ for all $i$ and $j$. Define a new basis for $F, x_{1}^{\prime}, x_{2}^{\prime}, \ldots, x_{n}^{\prime}$, from the old basis $x_{1}, x_{2}, \ldots, x_{n}$ by $x_{1}^{\prime}=x_{1}-\sum_{j>2} p x_{j}$ and $x_{i}^{\prime}=x_{i}$ for $i=$ $2,3, \ldots, n$. Thus

$$
s_{i}=b_{1}^{i} x_{1}^{\prime}+\sum_{j>2}\left(b_{j}^{i}+p b_{1}^{i}\right) x_{j}^{\prime}
$$

and hence

$$
S \subseteq\left\langle x_{1}^{\prime}, x_{2}^{\prime}, \ldots, x_{n}^{\prime}\right\rangle .
$$

THEOREM 3. If $S$ is a finitely generated totally cancellative reduced semigroup, then $G(\alpha(S))$ is pure in $G(F(S))$, the universal group of the free envelope $(f(S), \alpha)$ of $S$.

Proof. Let $U$ denote the subgroup of $G(F(S))$ generated by $\alpha(S)$. Let $P$ denote the pure subgroup of $G(F(S))$ generated by $\alpha(S)$. Denote $P \cap F(S)$ by $F$. Now $U \subseteq P$, and let us assume that $P \neq U$. Then

(1) There exists a basis of $P, x_{1}, x_{2}, \ldots, x_{n}$ such that $\alpha(S) \subseteq$ $\left\langle x_{1}, x_{2}, \ldots, x_{n}\right\rangle$, by Lemma 2 .

(2) There exists a surjective homomorphism $\beta: P \rightarrow Z$ such that $\beta_{\mid U}$ is not surjective and $\beta\left(x_{i}\right)>0$ for all $i$, by Lemma 1 .

Let $c$ be the smallest positive integer in the set $\beta(U)$. Then by (2), $c>1$. Thus define $\beta^{\prime}(a)=\beta(a) / c$ for all $a \in U$. Let $\beta^{\prime \prime}=\beta^{\prime} \mid S$. Then by (1) and (2), $\beta, \beta^{\prime}$ and $\beta^{\prime \prime}$ are all positive on $S \backslash\{0\}$. Since $\beta^{\prime}$ is the unique extension of $\beta^{\prime \prime}$ to $U$, and $\beta^{\prime}$ has a unique extension to $\gamma: P \rightarrow Q$ (as $x \in P$ implies $n x \in U$ for some $n \in N$ ). Since $\beta^{\prime}=\beta / c$ on $U$, then $\gamma=\beta / c$, and because $c>1$ and $\operatorname{gcd}(\beta(P))=1, \gamma$ must have nonintegral values. Therefore, $\beta^{\prime}$ cannot be extended to $P$ (as an integral valued homomorphism). On the other hand, the quasi-universal property of free envelopes says that $\beta^{\prime \prime}$ and hence $\beta^{\prime}$ can be extended to $F(S)$ and thus to $G(F(S)) \supseteq P$, a contradiction. Therefore, $U=P$, and hence $U$ is pure in $G(F(S))$ which completes the proof of the theorem.

A subsemigroup $S$ of a semigroup $K$ is said to be unitary if $x+a \in S$ and $a \in S$ implies $x \in S$ for all $x, a \in S$. 
DEFINITION. If $S$ is a subsemigroup of a semigroup $K$, then the unitary closure of $S$ with respect to $K, U_{k}(S)$, is the smallest unitary subsemigroup of $K$ containing $S$. Note: If $S$ is a finitely generated totally cancellative reduced semigroup which is contained in a finitely generated free semigroup $F$, then $U_{k}(S)=G(S) \cap F$.

Definition. Let $S$ be a subsemigroup of a cancellative semigroup $K$, and let $P$ be the pure subgroup of $G(K)$ generated by $S$. Then the pure unitary closure of $S$ in $K, P_{k}(S)$, is $P \cap K$.

COROLlARY 4. If $S$ is a finitely generated totally cancellative reduced semigroup, then the unitary closure of $\alpha(S)$ in the free envelope $(F(S), \alpha)$ of $S$ coincides with the pure unitary closure of $\alpha(S)$ in $F(S)$.

Proof. This follows directly from the theorem.

Definition. Let $S$ be a semigroup, and $\mathbf{N}$ be the positive integers under addition. Then the dual of $S, S^{*}$, is equal to $\operatorname{Hom}\left(S, N^{0}\right)$. From [1] it is known that:

(1) $S^{*} \simeq S^{* * *}$;

(2) The evaluation map $\sigma: S \rightarrow S^{* *}$ is injective if $S$ is finitely generated totally cancellative reduced;

(3) If $f: S^{* *} \rightarrow T$ is injective when restricted to $\sigma(S)$, then $f$ is injective;

(4) If $(F(S), \alpha)$ is the free envelope of $S$, then there exists an isomorphism, $K$, such that the following diagram commutes:

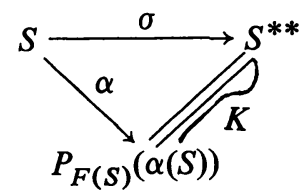

(5) If $f: S^{* *} \rightarrow T$ is a homomorphism and $f \mid \sigma(S)$ is an epimorphism, then $f$ is an epimorphism.

COROllary 5. If $S$ is a finitely generated totally cancellative reduced semigroup, then $S^{* *} \simeq U_{F(S)}(\alpha(S))$.

Proof. The proof follows directly from Corollary 4 and Proposition 3.7 of [1].

COROLlary 6. Let $S$ be a finitely generated totally cancellative reduced semigroup. Then $S \simeq S^{* *}$. If and only if $S$ can be embedded as a unitary subsemigroup of a finitely generated free semigroup.

Proof. Let $S \simeq S^{* *}$. Then by Corollary $4, S \simeq S^{* *} U_{F(S)}(\alpha(S))$, and $U_{F(S)}(\alpha(S))$ is unitary in $F(S)$.

Conversely, assume that $S$ is a unitary subsemigroup of a finitely generated free semigroup $F$. Then by hypothesis $S=\bar{S}_{F}$. Therefore, there exists a homomorphism $f: F(S) \rightarrow F$ such that the following diagram commutes: 


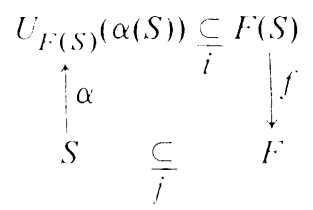

Hence $f \alpha=1_{S}$.

If $x \in F(S), \alpha(s) \in \alpha(S)$ and $x+\alpha(s) \in \alpha(S)$, then $x \in U_{F(S)}(\alpha(S))$. Hence $a+x \in \alpha(S)$ for some $a \in \alpha(S)$. Thus $f(a)+f(x) \in S$. Therefore, $f(s) \in S$. Thus $U_{F(S)}(\alpha(S)) \subseteq S$. Thus there is an induced homomorphism $g$ : $U_{F(S)}(\alpha(S)) \rightarrow S$ induced by the following commutative diagram:

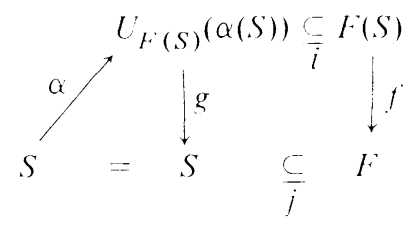

Therefore, $g \alpha=1_{S}$ and also $\alpha g \alpha=\alpha=1_{U_{F(S)}}(\alpha(S)) \alpha$. Thus $\alpha g=$ $1_{U_{F(S)}}(\alpha(S))$, by property (5) above. Therefore, by Corollary $4, S=$ $U_{F(S)}(\alpha(S)) \cong S^{* *}$.

\section{REFERENCES}

1. P. A. Grillet, The free envelope of a finitely generated commutative semigroup, Trans. Amer. Math. Soc. 149 (1970), 665-682. MR 45 \#2056.

2. A. H. Clifford and G. B. Preston, The algebraic theory of semigroups. Vols. 1, 2, Math. Surveys, no. 7, Amer. Math. Soc., Providence, R. I., 1961, 1967. MR 24 \# A2627; 36 \#1558.

3. L. Fuchs, Infinite abelian groups. Vol. 1., Academic Press, New York, 1970. MR 41 \#33.

ibM Corporation, Department D89, Building 705-2, P. O. Box 390, Poughkeepsie, New YORK 12602 